\title{
A Note on the Adaptive Estimation of a Conditional Continuous-Discrete Multivariate Density by Wavelet Methods
}

\author{
Christophe Chesneau ${ }^{1}$ and Hassan Doosti ${ }^{2}$ \\ ${ }^{1}$ Laboratoire de Mathématiques Nicolas Oresme, Université de Caen, BP 5186, 14032 Caen Cedex, France \\ ${ }^{2}$ Mashhad University of Medical Sciences, P.O. Box 91735-951, Mashhad, Iran \\ Correspondence should be addressed to Christophe Chesneau; christophe.chesneau@gmail.com
}

Received 11 April 2016; Revised 24 May 2016; Accepted 6 June 2016

Academic Editor: Niansheng Tang

Copyright (c) 2016 C. Chesneau and H. Doosti. This is an open access article distributed under the Creative Commons Attribution License, which permits unrestricted use, distribution, and reproduction in any medium, provided the original work is properly cited.

We investigate the estimation of a multivariate continuous-discrete conditional density. We develop an adaptive estimator based on wavelet methods. We prove its good theoretical performance by determining sharp rates of convergence under the $\mathbb{L}_{p}$ risk with $p \geq 1$ for a wide class of unknown conditional densities. A simulation study illustrates the good practical performance of our estimator.

\section{Introduction}

The estimation of conditional densities is an important statistical challenge with applications in many practical problems, especially those connected with forecasting (economics, etc.). There is a vast literature in this area. We refer to the papers of Li and Racine [1], Akakpo and Lacour [2], and Chagny [3] and the references therein. In this paper we focus our attention on a specific problem: the estimation of a multivariate continuous-discrete conditional density. The considered model is described as follows. Let $d, d_{*}, v$, and $n$ be positive integers and let $\left(\mathbf{X}_{1}, \mathbf{Y}_{1}\right), \ldots,\left(\mathbf{X}_{n}, \mathbf{Y}_{n}\right)$ be $n$ iid random vectors defined on the probability space $(\Omega, \mathscr{A}, P)$. We suppose that $\mathbf{X}_{1}$ is continuous with support $[0,1]^{d}$ and that $\mathbf{Y}_{1}$ is discrete with support $\{0,1, \ldots, \nu\}^{d_{*}}$. Let $f$ be the density of $\left(\mathbf{X}_{1}, \mathbf{Y}_{1}\right)$. We define the density function of $\mathbf{X}_{1}$ conditionally to the event $\{\mathbf{Y}=\mathbf{m}\}$ by

$$
g(\mathbf{x}, \mathbf{m})=f\left(\mathbf{x} \mid \mathbf{Y}_{1}=\mathbf{m}\right)=\frac{f(\mathbf{x}, \mathbf{m})}{P\left(\mathbf{Y}_{1}=\mathbf{m}\right)}
$$

$(\mathbf{x}, \mathbf{m}) \in[0,1]^{d} \times\{0,1, \ldots, \nu\}^{d_{*}}$. We aim to estimate $g(\mathbf{x}, \mathbf{m})$ from $\left(\mathbf{X}_{1}, \mathbf{Y}_{1}\right), \ldots,\left(\mathbf{X}_{n}, \mathbf{Y}_{n}\right)$. The most common approach is based on the kernel methods developed by Li and Racine [4].
Applications and recent developments for these methods are described in detail in Li and Racine [1].

In this paper we develop a new estimator $\hat{g}(\mathbf{x}, \mathbf{m})$ based on wavelet methods. It is now an established fact that, in comparison to kernel methods, wavelet methods have the advantage to achieve a high degree of adaptivity for a large class of unknown functions, with possible complex discontinuities (jumps, spikes, etc.). See, for instance, Antoniadis [5], Härdle et al. [6], and Vidakovic [7]. This fact motivates our interest to develop wavelet methods for the considered conditional density estimation problem. The main ingredients in the construction of $\hat{g}(\mathbf{x}, \mathbf{m})$ are an estimation of $f(\mathbf{x}, \mathbf{m})$ with a new wavelet estimator $\hat{f}(\mathbf{x}, \mathbf{m})$, an estimation of $P\left(\mathbf{Y}_{1}=\right.$ $\mathbf{m})$ by an empirical estimator, and a global thresholding technique developed by Vasiliev [8]. In particular, the considered estimator $\widehat{f}(\mathbf{x}, \mathbf{m})$ can be viewed as a multivariate (but "nonsmooth") version of the one introduced in the univariate case, that is, $d=d_{*}=1$, in Chesneau et al. [9]. We prove that $\hat{g}(\mathbf{x}, \mathbf{m})$ is both adaptive and efficient; it is not dependent on the smoothness of $g(\mathbf{x}, \mathbf{m})$ in its construction and, under mild assumptions on the smoothness of $g(\mathbf{x}, \mathbf{m})$ (we assume that it belongs to a wide class of functions, the so-called Besov balls), it attains fast rates of convergence under the $\mathbb{L}_{p}$ risk (with $p \geq 1$ ). These theoretical guarantees are illustrated 
by a numerical study showing the good practical performance of our estimator.

The remainder of this paper is set out as follows. Next, in Section 2, we briefly describe the considered multidimensional wavelet bases and Besov balls. Our wavelet estimator and some of its theoretical properties are presented in Section 3. A short numerical study can be found in Section 4. Finally, the proofs are postponed to Section 5.

\section{Multidimensional Wavelet Bases and Besov Balls}

Let $d$ be positive integers and let $p \geq 1$. First of all, we define the $\mathbb{L}_{p}\left([0,1]^{d}\right)$ spaces as $\mathbb{L}_{p}\left([0,1]^{d}\right)=\left\{f:[0,1]^{d} \rightarrow\right.$ $\left.\mathbb{R} ; \int_{[0,1]^{d}}|f(\mathbf{x})|^{p} d \mathbf{x}<\infty\right\}$.

In this study, we consider a wavelet base on $[0,1]^{d}$ based on the scaling and wavelet functions $\phi$ and $\psi$, respectively, from Daubechies family (see [10]). For any $\mathbf{x}=\left(x_{1}, \ldots, x_{d}\right) \in$ $[0,1]^{d}$, we set

$$
\begin{aligned}
& \Phi(\mathbf{x})=\prod_{v=1}^{d} \phi\left(x_{v}\right), \\
& \Psi_{u}(\mathbf{x}) \\
& = \begin{cases}\psi\left(x_{u}\right) \prod_{\substack{v=1 \\
v \neq u}}^{d} \phi\left(x_{v}\right), & \text { for } u \in\{1, \ldots, d\}, \\
\prod_{v \in A_{u}} \psi\left(x_{v}\right) \prod_{v \notin A_{u}} \phi\left(x_{v}\right), & \text { for } u \in\left\{d+1, \ldots, 2^{d}-1\right\},\end{cases}
\end{aligned}
$$

where $\left(A_{u}\right)_{u \in\left\{d+1, \ldots, 2^{d}-1\right\}}$ forms the set of all nonvoid subsets of $\{1, \ldots, d\}$ of cardinality greater than or equal to 2 .

For any integer $j$ and any $\mathbf{k}=\left(k_{1}, \ldots, k_{d}\right)$, we consider

$$
\begin{gathered}
\Phi_{j, \mathbf{k}}(\mathbf{x})=2^{j d / 2} \Phi\left(2^{j} x_{1}-k_{1}, \ldots, 2^{j} x_{d}-k_{d}\right), \\
\Psi_{j, \mathbf{k}, u}(\mathbf{x})=2^{j d / 2} \Psi_{u}\left(2^{j} x_{1}-k_{1}, \ldots, 2^{j} x_{d}-k_{d}\right),
\end{gathered}
$$

$$
\text { for any } u \in\left\{1, \ldots, 2^{d}-1\right\} \text {. }
$$

Let $\mathbf{D}_{j}=\left\{0, \ldots, 2^{j}-1\right\}^{d}$. Then, with an appropriate treatment at the boundaries, there exists an integer $\tau$ such that the collection $\left\{\Phi_{\tau, \mathbf{k}}, \mathbf{k} \in \mathbf{D}_{\tau} ;\left(\Psi_{j, \mathbf{k}, u}\right)_{u \in\left\{1, \ldots, 2^{d}-1\right\}}, j \in\right.$ $\left.\mathbb{N}-\{0, \ldots, \tau-1\}, \mathbf{k} \in \mathbf{D}_{j}\right\}$ forms an orthonormal basis of $\mathbb{L}_{2}\left([0,1]^{d}\right)$. A function $f \in \mathbb{L}_{2}\left([0,1]^{d}\right)$ can be expanded into a wavelet series as

$$
\begin{array}{r}
f(\mathbf{x})=\sum_{\mathbf{k} \in \mathbf{D}_{\tau}} c_{\tau, \mathbf{k}} \Phi_{\tau, \mathbf{k}}(\mathbf{x})+\sum_{u=1}^{2^{d}-1} \sum_{j=\tau}^{\infty} \sum_{\mathbf{k} \in \mathbf{D}_{j}} d_{j, \mathbf{k}, u} \Psi_{j, \mathbf{k}, u}(\mathbf{x}), \\
\mathbf{x} \in[0,1]^{d},
\end{array}
$$

where

$$
\begin{aligned}
c_{\tau, \mathbf{k}} & =\int_{[0,1]^{d}} f(\mathbf{x}) \Phi_{\tau, \mathbf{k}}(\mathbf{x}) d \mathbf{x}, \\
d_{j, \mathbf{k}, u} & =\int_{[0,1]^{d}} f(\mathbf{x}) \Psi_{j, \mathbf{k}, u}(\mathbf{x}) d \mathbf{x} .
\end{aligned}
$$

All the details about these wavelet bases, including the expansion into wavelet series as described above, can be found in, for example, Meyer [11], Daubechies [10], Cohen et al. [12], and Mallat [13].

Let $M>0, s \in(0, N), p \geq 1$, and $r \geq 1$. We say that a function $f \in \mathbb{L}_{2}\left([0,1]^{d}\right)$ belongs to the Besov ball $\mathbf{B}_{r, q}^{s}(M)$ if and only if the associated wavelet coefficients (5) satisfy

$$
\begin{aligned}
& \left(\sum_{\mathbf{k} \in \mathbf{D}_{\tau}}\left|c_{\tau, \mathbf{k}}\right|^{r}\right)^{1 / r} \\
& +\left(\sum_{j=\tau}^{\infty}\left(2^{j(s+d(1 / 2-1 / r))}\left(\sum_{u=1}^{2^{d}-1} \sum_{\mathbf{k} \in \mathbf{D}_{j}}\left|d_{j, \mathbf{k}, u}\right|^{r}\right)^{1 / r}\right)^{q}\right)^{1 / q}
\end{aligned}
$$$$
\leq M
$$

and with the usual modifications for $r=\infty$ or $q=\infty$.

These sets contain function classes of significant spatial inhomogeneity, including Sobolev balls and Hölder balls. Details about Besov balls can be found in, for example, Meyer [11] and Härdle et al. [6].

\section{Conditional Density Estimation}

We formulate the following assumptions.

(B1) There exists a known constant $C>0$ such that

$$
\sup _{\mathbf{x} \in[0,1]^{d}} \sup _{\mathbf{m} \in\{0,1, \ldots, \nu\}^{d *}} f(\mathbf{x}, \mathbf{m}) \leq C .
$$

(B2) There exists a known constant $c \in(0,1)$ such that

$$
c \leq \inf _{\mathbf{m} \in\{0,1, \ldots, v\}^{d_{*}}} P\left(\mathbf{Y}_{1}=\mathbf{m}\right) .
$$

We propose the following "ratio-thresholding estimator" $\widehat{g}(\mathbf{x}, \mathbf{m})$ for $g(\mathbf{x}, \mathbf{m})$ :

$$
\widehat{g}(\mathbf{x}, \mathbf{m})=\frac{\widehat{f}(\mathbf{x}, \mathbf{m})}{\widehat{\rho}_{\mathbf{m}}} \mathbf{1}_{\left\{\hat{\rho}_{\mathbf{m}} \geq c / 2\right\}},
$$

$(\mathbf{x}, \mathbf{m}) \in[0,1]^{d} \times\{0,1, \ldots, \nu\}^{d_{*}}$, where 1 denotes the indicator function, $c$ refers to the constant in (B2), and $\widehat{f}(\mathbf{x}, \mathbf{m})$ is defined by

$$
\begin{aligned}
& \widehat{f}(\mathbf{x}, \mathbf{m})=\sum_{\mathbf{k} \in \mathbf{D}_{\tau}} \widehat{c}_{\tau, \mathbf{k}}(\mathbf{m}) \Phi_{\tau, \mathbf{k}}(\mathbf{x}) \\
& \quad+\sum_{u=1}^{2^{d}-1} \sum_{j=\tau}^{j_{1}} \sum_{\mathbf{k} \in \mathbf{D}_{j}} \widehat{d}_{j, \mathbf{k}, u}(\mathbf{m}) \mathbf{1}_{\left\{\left|\widehat{d}_{j, \mathbf{k}, u}(\mathbf{m})\right| \geq \kappa \sqrt{\ln (n) / n\}}\right.} \Psi_{j, \mathbf{k}, u}(\mathbf{x}),
\end{aligned}
$$

where

$$
\begin{gathered}
\widehat{c}_{\tau, \mathbf{k}}(\mathbf{m})=\frac{1}{n} \sum_{i=1}^{n} \Phi_{\tau, \mathbf{k}}\left(\mathbf{X}_{i}\right) \mathbf{1}_{\left\{\mathbf{Y}_{i}=\mathbf{m}\right\}}, \\
\widehat{d}_{j, \mathbf{k}, u}(\mathbf{m})=\frac{1}{n} \sum_{i=1}^{n} \Psi_{j, \mathbf{k}, u}\left(\mathbf{X}_{i}\right) \mathbf{1}_{\left\{\mathbf{Y}_{i}=\mathbf{m}\right\}},
\end{gathered}
$$


$\kappa$ is a large enough constant and $j_{1}$ is an integer such that $n / \ln (n) \leq 2^{j_{1} d} \leq 2 n / \ln (n)$, and $\hat{\rho}_{\mathbf{m}}$ is defined by

$$
\widehat{\rho}_{\mathbf{m}}=\frac{1}{n} \sum_{i=1}^{n} \mathbf{1}_{\left\{\mathbf{Y}_{i}=\mathbf{m}\right\}} .
$$

The estimator (10) uses a hard thresholding technique of the wavelet coefficients estimators (12). Such a selection rule is at the heart of the adaptive nature of wavelet methods which have the ability to capture the most important wavelet coefficients of a function, that is, those with the high magnitudes. We refer to Antoniadis [5], Härdle et al. [6], and Vidakovic [7] for further details. The definition of the threshold, that is, $\lambda_{n}=\kappa \sqrt{\ln (n) / n}$, corresponds to the universal one proposed by Donoho and Johnstone [14] and Donoho et al. [15]. It is based on technical considerations ensuring good convergence properties of the hard thresholding wavelet estimator (see also Theorem A.3 in Appendix).

Note that (10) can be viewed as a nonsmooth multivariate version of the estimator proposed by Chesneau et al. [9]. The main advantage of this estimator is to be more easy to implement from a practical point of view (see Section 4

$$
\Theta_{n}= \begin{cases}\left(\frac{\ln (n)}{n}\right)^{s p /(2 s+d)}, & \text { for } 2 r s>d(p-r), \\ \left(\frac{\ln (n)}{n}\right)^{(s-d(1 / r-1 / p)) p /(2 s-2 d / r+d)}, & \text { for } 2 r s<d(p-r), \\ \left(\frac{\ln (n)}{n}\right)^{(s-d(1 / r-1 / p)) p /(2 s-2 d / r+d)}(\ln (n))^{(p-2 r / q)_{+}}, & \text {for } 2 r s=d(p-r) .\end{cases}
$$

The proof of Theorem 1 is based on several technical inequalities and the application of a general result derived from [16, Theorem 5.1] and [17, Theorem 1] (see Theorem A.3 in Appendix).

Theorem 1 provides theoretical guarantees on the convergence of (9) under mild assumptions on the smoothness of $f(\mathbf{x}, \mathbf{m})$ and a fortiori $g(\mathbf{x}, \mathbf{m})$ under the $\mathbb{L}_{p}$ risk. The obtained rates of convergence are sharp. However, since the lower minimax bounds are not established in our setting, we do not claim that they are the optimal ones in the minimax sense. An important benchmark is that they correspond to the optimal ones in the minimax sense for the standard multivariate density estimation problem, corresponding to $d_{*}=1$ and $\mathbf{Y}_{1}$, is constant almost surely, up to a logarithmic term (see [15]).

Finally, note that the factor $d_{*}$ plays a secondary role in our study; it only appears in the presentation of the model and the construction of $\widehat{\rho}_{\mathbf{m}}$ and its performance does not depend on the value of $d_{*}$.

\section{A Short Numerical Study}

In this section we investigate some practical aspects of our wavelet methods. For the sake of simplicity, we focus our attention on the univariate case, that is, $d=d_{*}=1$ (so $\mathbf{x}=x, \mathbf{m}=m, \mathbf{Y}_{1}=Y$, etc.). The codes are written below for a numerical comparison in the univariate case). Concerning $\widehat{\rho}_{n}$, let us mention that it is a natural unbiased estimator for $P\left(\mathbf{Y}_{1}=\mathbf{m}\right)$ with nice convergence properties. They will be used in the proof of our main result.

The global construction of (9) follows the idea proposed by Vasiliev [8] for other statistical contexts. Note that a control on the lower bound of $\hat{\rho}_{\mathbf{m}}$ is necessary; it must be large enough to ensure good statistical properties for (9).

The following result investigates the rates of convergence attained by (9) under the $\mathbb{L}_{p}$ risk with $p \geq 1$.

Theorem 1. Let $\mathbf{m} \in\{0,1, \ldots, \nu\}^{d_{*}}$, let $p \geq 1$, let $g(\mathbf{x}, \mathbf{m})$ be (1), and let $\hat{g}(\mathbf{x}, \mathbf{m})$ be defined by (9) with a large enough $\kappa$ (the exact condition is described in (29)). Suppose that (B1) and (B2) hold and that $f(\mathbf{x}, \mathbf{m}) \in \mathbf{B}_{r, q}^{s}(M)$ with $M>0, s>d / r$, $r \geq 1$, and $q \geq 1$. Then there exists a constant $C>0$ such that, for $n$ being large enough,

$$
E\left(\int_{[0,1]^{d}}|\widehat{g}(\mathbf{x}, \mathbf{m})-g(\mathbf{x}, \mathbf{m})|^{p} d \mathbf{x}\right) \leq C \Theta_{n},
$$

where

in MATLAB and are adapted from Ramirez and Vidakovic [18]. First we compare the performance of new estimators of density functions $f(x, m)$ with those proposed in our former publication, Chesneau et al. [9], in two styles, accuracy and speed of computation. In order to illustrate the rate of decrease of errors, as Chesneau et al. [9], we employ the indicator defined by

$$
L_{2} \text {-Norm }=\frac{1}{100 n N} \sum_{i=1}^{N} \sum_{j=1}^{n}\left(\widehat{f}_{i}\left(\frac{j}{n}\right)-f\left(\frac{j}{n}\right)\right)^{2}
$$

where $n$ and $N$ are sample size and the number of replications, respectively, $f$ represents the true density, and $\widehat{f}$ is an estimator. We consider three estimators based on our statistical methodology: the linear wavelet estimator; that is,

$$
\widehat{f}_{L}(x, m)=\sum_{k=0}^{2^{j_{0}}-1} \widehat{c}_{j_{0}, k}(m) \phi_{j_{0}, k}(x),
$$

$x \in[0,1]$, the hard thresholding wavelet estimator defined by (10), and the smooth version of the linear wavelet estimator after local linear regression (see, e.g., [19]). The practical construction of this smooth version of linear wavelet estimators was proposed by Ramirez and Vidakovic [18]. 
TABLE 1: Computed values for $L_{2}$-Norm for various sample sizes.

\begin{tabular}{lccccccc}
\hline \multirow{2}{*}{ Estimator } & \multirow{2}{*}{ Density } & $n=20$ & $n=50$ & $n=100$ & $n=200$ & $n=500$ \\
\hline Linear & $f(x, 0)$ & 0.0188 & 0.0080 & 0.0038 & 0.0019 & 0.00080 \\
Linear & $f(x, 1)$ & 0.0132 & 0.0050 & 0.0027 & 0.0013 & 0.00051 \\
\hline Hard thresh. & $f(x, 0)$ & 0.0178 & 0.0073 & 0.0033 & 0.0016 & 0.0006 & 0.00026 \\
Hard thresh. & $f(x, 1)$ & 0.0123 & 0.0044 & 0.0021 & 0.00096 & 0.00036 \\
Smooth & $f(x, 0)$ & 0.0011 & 0.00047 & 0.00026 & 0.00017 & 0.00012 \\
Smooth & $f(x, 1)$ & 0.00063 & 0.00028 & 0.00014 & 0.00007 & 0.00004 & 0.000017 \\
\hline
\end{tabular}

TABLE 2: Elapsed time (seconds).

\begin{tabular}{lcccccc}
\hline \multirow{2}{*}{ Estimator } & \multicolumn{4}{c}{ Sample size } \\
& $n=20$ & $n=50$ & $n=100$ & $n=200$ & $n=500$ & $n=1000$ \\
\hline Chesneau et al. [9] & 64.3205 & 157.546 & 176.094 & 369.802 & 771.294 & 2277.76 \\
New estimators & 0.641954 & 0.89170 & 1.481340 & 2.62647 & 5.78828 & 11.267064 \\
\hline
\end{tabular}

Several studies confirm that this version of estimators has nice performance in different fields (see, e.g., [20, 21]). We adopt similar setup from Chesneau et al. [9] for our example; that is, we use Daubechies's compactly supported "Daubechies 3" and we take $j_{0}=6$. Also, we generate different sample sizes $n=20,50,100,200,500$, and 1000 data points $X_{1}, \ldots, X_{n}$, from $\operatorname{Beta}(2,3)$ distribution. The discrete random sample is generated from $\operatorname{Binomial}\left(1, x_{i}\right)$; the bivariate density function is

$$
f(x, m)=12 x^{1+m}(1-x)^{3-m},
$$

$(x, m) \in[0,1] \times\{0,1\}$. Table 1 gives the value of $L_{2}$-Norm computed from 100 simulations for different sample sizes. This table should be compared with Table 1 in page 70 in Chesneau et al. [9]. As we see, similar results could be obtained; $L_{2}$-Norm decreases while the sample size increases. The performance of the smooth version of linear wavelet estimator is the best. As we see there is no significant difference between the new version of estimators with former versions in Chesneau et al. [9].

On the other hand, Table 2 depicts the speed of computation for two groups of estimators in seconds. The codes are run with an ordinary laptop with 4.3 RAM. As we see the speed of new version of estimators is much less than the former. For example, when the sample size is 1000 , the speed of computation is about 200 times less than the former version of wavelets estimators of densities. These differences will be much bigger when the sample size increases.

In the second part of this section we show the performance of proposed estimators of conditional density functions. Note that the conditional density function in the above examples satisfies

$$
\begin{aligned}
g(x, m) & =f(x \mid Y=m) \\
& = \begin{cases}20 x(1-x)^{3}, & \text { for } m=0, \\
30 x^{2}(1-x)^{2}, & \text { for } m=1,\end{cases}
\end{aligned}
$$

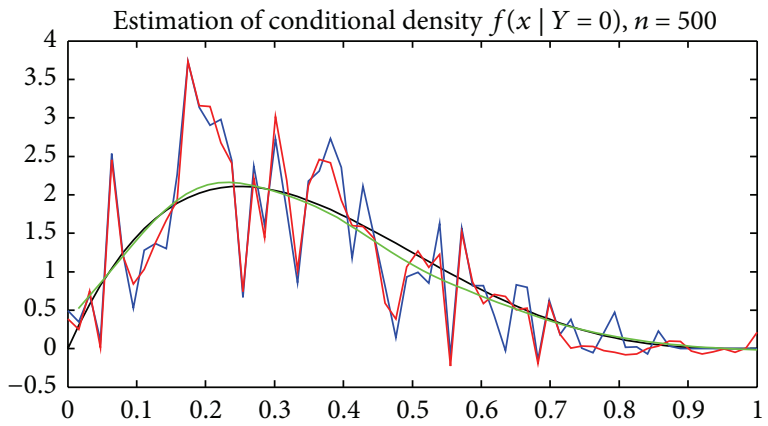

FIGURE 1: The true conditional density function $g(x, 0)$ is shown in black line, the wavelet linear estimator is blue, the wavelet hard thresholding estimator is red, and its smooth version is green with $n=500$.

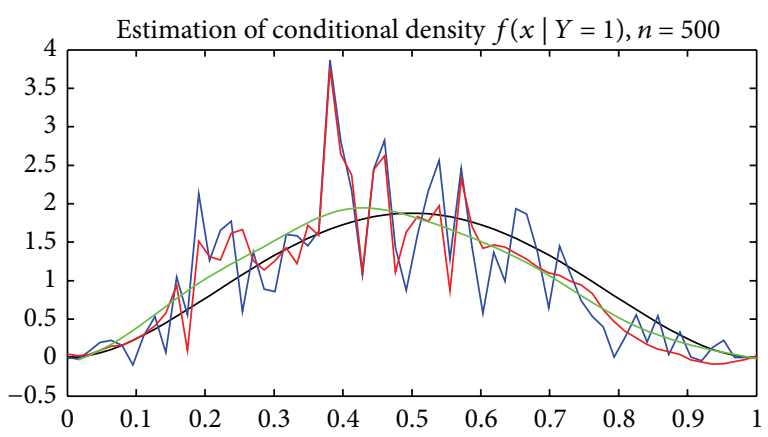

FIGURE 2: The true conditional density function $g(x, 1)$ is shown in black line, the wavelet linear estimator is blue, the wavelet hard thresholding estimator is red, and its smooth version is green with $n=500$.

$x \in[0,1]$. Figures 1 and 2 depict $g(x, 0)$ and $g(x, 1)$, respectively. In each case the true conditional density function is shown in black line, the linear wavelet estimator is blue, the hard thresholding wavelet estimator is red, and the smooth version of linear one is green. 
TABLE 3: Computed values for $100 L_{2}$-Norm for various sample sizes.

\begin{tabular}{|c|c|c|c|c|c|c|c|}
\hline \multirow{2}{*}{ Estimator } & \multirow{2}{*}{ Conditional density } & \multicolumn{6}{|c|}{ Sample size } \\
\hline & & $n=20$ & $n=50$ & $n=100$ & $n=200$ & $n=500$ & $n=1000$ \\
\hline Linear & $g(x, 0)$ & 5.3407 & 1.1357 & 1.0883 & 0.5421 & 0.2150 & 0.1131 \\
\hline Linear & $g(x, 1)$ & 8.8589 & 3.2498 & 1.6118 & 0.7908 & 0.3255 & 0.1614 \\
\hline Hard thresh. & $g(x, 0)$ & 5.0716 & 1.9346 & 0.9430 & 0.4576 & 0.1745 & 0.0900 \\
\hline Hard thresh. & $g(x, 1)$ & 8.1867 & 2.7903 & 1.2612 & 0.5851 & 0.2287 & 0.1064 \\
\hline Smooth & $g(x, 0)$ & 0.2303 & 0.1064 & 0.0682 & 0.0433 & 0.0286 & 0.0261 \\
\hline Smooth & $g(x, 1)$ & 0.3351 & 0.1297 & 0.0684 & 0.0377 & 0.0196 & 0.0145 \\
\hline
\end{tabular}

All the figures illustrate the good performance of our proposed linear and nonlinear estimators of conditional density functions. It should be reminded that the hard thresholding one has no tuning parameter; it is entirely adaptive. The smooth version of our wavelet linear estimator has the best performance. Furthermore, Table 3 represents the impact of sample size on performance of our estimators. This table also compares the performance of three estimators. The number of replications is 500 . As the sample size increases the value of indicator decreases and the performance of smooth version of linear wavelet estimators is the best.

\section{Proof of Theorem 1}

In what follows, $C$ denotes any constant that does not depend on $j, \mathbf{k}$, and $n$. Its value may change from one term to another. For the sake of simplicity, we set $\rho_{\mathbf{m}}=P\left(\mathbf{Y}_{1}=\mathbf{m}\right)$. Observe that

$$
\begin{aligned}
\hat{g} & (\mathbf{x}, \mathbf{m})-g(\mathbf{x}, \mathbf{m})=\frac{\hat{f}(\mathbf{x}, \mathbf{m})}{\hat{\rho}_{\mathbf{m}}} \mathbf{1}_{\left\{\hat{\rho}_{\mathbf{m}} \geq c / 2\right\}}-\frac{f(\mathbf{x}, \mathbf{m})}{\rho_{\mathbf{m}}} \\
= & \frac{1}{\hat{\rho}_{\mathbf{m}} \rho_{\mathbf{m}}}\left(\rho_{\mathbf{m}}(\hat{f}(\mathbf{x}, \mathbf{m})-f(\mathbf{x}, \mathbf{m}))\right. \\
& \left.+f(\mathbf{x}, \mathbf{m})\left(\rho_{\mathbf{m}}-\hat{\rho}_{\mathbf{m}}\right)\right) \mathbf{1}_{\left\{\hat{\rho}_{\mathbf{m}} \geq c / 2\right\}}-\frac{f(\mathbf{x}, \mathbf{m})}{\rho_{\mathbf{m}}} \\
& \cdot \mathbf{1}_{\left\{\hat{\rho}_{\mathbf{m}}<c / 2\right\}} .
\end{aligned}
$$

Owing to (B2), we have $\left\{\widehat{\rho}_{\mathbf{m}}<c / 2\right\} \subseteq\left\{\left|\widehat{\rho}_{\mathbf{m}}-\rho_{\mathbf{m}}\right|\right.$ $>c / 2\}$ implying that $\mathbf{1}_{\left\{\hat{\rho}_{\mathbf{m}}<c / 2\right\}} \leq(2 / c)\left|\hat{\rho}_{\mathbf{m}}-\rho_{\mathbf{m}}\right|$ and $\left(1 /\left(\hat{\rho}_{\mathbf{m}} \rho_{\mathbf{m}}\right)\right) \mathbf{1}_{\left\{\hat{\rho}_{\mathrm{m}} \geq c / 2\right\}} \leq 2 / c^{2}$. Moreover, note that $\rho_{\mathbf{m}} \leq 1$ and, thanks to $(\mathbf{B 1}), f(\mathbf{x}, \mathbf{m}) \leq C$. It follows from the triangular inequality and the above inequalities that

$$
\begin{aligned}
& |\hat{g}(\mathbf{x}, \mathbf{m})-g(\mathbf{x}, \mathbf{m})| \\
& \quad \leq C\left(|\hat{f}(\mathbf{x}, \mathbf{m})-f(\mathbf{x}, \mathbf{m})|+\left|\hat{\rho}_{\mathbf{m}}-\rho_{\mathbf{m}}\right|\right) .
\end{aligned}
$$

By the inequality $|x+y|^{p} \leq 2^{p-1}\left(|x|^{p}+|y|^{p}\right),(x, y) \in \mathbb{R}^{2}$, we obtain

$$
E\left(\int_{[0,1]^{d}}|\widehat{g}(\mathbf{x}, \mathbf{m})-g(\mathbf{x}, \mathbf{m})|^{p} d \mathbf{x}\right) \leq C(S+T),
$$

where

$$
\begin{aligned}
& S=E\left(\int_{[0,1]^{d}}|\widehat{f}(\mathbf{x}, \mathbf{m})-f(\mathbf{x}, \mathbf{m})|^{p} d \mathbf{x}\right), \\
& T=E\left(\left|\hat{\rho}_{\mathbf{m}}-\rho_{\mathbf{m}}\right|^{p}\right) .
\end{aligned}
$$

Let us now bound $S$ and $T$ in turn.

Upper Bound for $S$. We investigate an upper bound for $S$ by using Theorem A.3 in Appendix. First of all, thanks to (B1) implying that $f(\mathbf{x}, \mathbf{m}) \in \mathbb{L}_{2}\left([0,1]^{d}\right)$, let us expand the density $f(\mathbf{x}, \mathbf{m})$ on the considered wavelet basis:

$$
\begin{aligned}
f(\mathbf{x}, \mathbf{m})= & \sum_{\mathbf{k} \in \mathbf{D}_{\tau}} c_{\tau, \mathbf{k}}(\mathbf{m}) \Phi_{\tau, \mathbf{k}}(\mathbf{x}) \\
& +\sum_{u=1}^{2^{d}-1} \sum_{j=\tau}^{j_{1}} \sum_{\mathbf{k} \in \mathbf{D}_{j}} d_{j, \mathbf{k}, u}(\mathbf{m}) \Psi_{j, \mathbf{k}, u}(\mathbf{x}),
\end{aligned}
$$

where $c_{\tau, \mathbf{k}}(\mathbf{m})=\int_{[0,1]^{d}} f(\mathbf{x}, \mathbf{m}) \Phi_{\tau, \mathbf{k}}(\mathbf{x}) d \mathbf{x}$ and $d_{j, \mathbf{k}, u}(\mathbf{m})=$ $\int_{[0,1]^{d}} f(\mathbf{x}, \mathbf{m}) \Psi_{j, \mathbf{k}, u}(\mathbf{x}) d \mathbf{x}$. Let us now prove that the wavelet coefficients estimators $\widehat{c}_{j, \mathbf{k}}(\mathbf{m})$ and $\widehat{d}_{j, \mathbf{k}, u}(\mathbf{m})$ satisfy Assumptions (C1) and (C2) of Theorem A.3.

First of all, observe that $\widehat{c}_{\tau, \mathbf{k}}(\mathbf{m})$ and $\widehat{d}_{j, \mathbf{k}, u}(\mathbf{m})$ are unbiased estimators for $c_{\tau, \mathbf{k}}(\mathbf{m})$ and $d_{j, \mathbf{k}, u}(\mathbf{m})$, respectively:

$$
\begin{aligned}
E & \left(\widehat{d}_{j, \mathbf{k}, u}(\mathbf{m})\right)=E\left(\Psi_{j, \mathbf{k}, u}\left(\mathbf{X}_{1}\right) \mathbf{1}_{\left\{\mathbf{Y}_{1}=\mathbf{m}\right\}}\right) \\
& =\sum_{\mathbf{v} \in\{0,1, \ldots, q\}^{d d_{*}}} \int_{[0,1]^{d}} \Psi_{j, \mathbf{k}, u}(\mathbf{x}) \mathbf{1}_{\{\mathbf{v}=\mathbf{m}\}} f(\mathbf{x}, \mathbf{v}) d \mathbf{x} \\
& =\int_{[0,1]^{d}} \Psi_{j, \mathbf{k}, u}(\mathbf{x}) f(\mathbf{x}, \mathbf{m}) d \mathbf{x}=d_{j, \mathbf{k}, u}(\mathbf{m}) .
\end{aligned}
$$

We prove similarly that $E\left(\widehat{c}_{\tau, \mathbf{k}}(\mathbf{m})\right)=c_{\tau, \mathbf{k}}(\mathbf{m})$.

Investigation of (C1). Let us focus on the second inequality in (C1); the first one can be proved with similar arguments. For any $i \in\{1, \ldots, n\}$, set $V_{i}=\Psi_{j, \mathbf{k}, u}\left(\mathbf{X}_{i}\right) \mathbf{1}_{\left\{\mathbf{Y}_{i}=\mathbf{m}\right\}}-d_{j, \mathbf{k}, u}(\mathbf{m})$. Then 
$V_{1}, \ldots, V_{n}$ are $n$ zero-mean iid random variables with, by $(\mathbf{B} 1)$ and $2^{d j} \leq 2 n$,

$$
\begin{aligned}
& E\left(\left|V_{1}\right|^{2 p}\right) \leq C E\left(\left|\Psi_{j, \mathbf{k}, u}\left(\mathbf{X}_{1}\right) \mathbf{1}_{\left\{\mathbf{Y}_{1}=\mathbf{m}\right\}}\right|^{2 p}\right) \\
& \quad=C \sum_{\mathbf{v} \in\{0,1, \ldots,, q\}^{d_{*}}} \int_{[0,1]^{d}}\left|\Psi_{j, \mathbf{k}, u}(\mathbf{x}) \mathbf{1}_{\{\mathbf{v}=\mathbf{m}\}}\right|^{2 p} f(\mathbf{x}, \mathbf{v}) d \mathbf{x} \\
& \quad=C \int_{[0,1]^{d}}\left|\Psi_{j, \mathbf{k}, u}(\mathbf{x})\right|^{2 p} f(\mathbf{x}, \mathbf{m}) d \mathbf{x} \\
& \quad \leq C \int_{[0,1]^{d}}\left|\Psi_{j, \mathbf{k}, u}(\mathbf{x})\right|^{2 p} d \mathbf{x} \\
& \quad \leq C 2^{j d(p-1)} \int_{[0,1]^{d}}\left|\Psi_{j, \mathbf{k}, u}(\mathbf{x})\right|^{2} d \mathbf{x} \leq C n^{p-1} .
\end{aligned}
$$

It follows from the Rosenthal inequality (see Appendix) that

$$
\begin{aligned}
& E\left(\left|\hat{d}_{j, \mathbf{k}, u}-d_{j, \mathbf{k}, u}\right|^{2 p}\right)=E\left(\left|\frac{1}{n} \sum_{i=1}^{n} V_{i}\right|^{2 p}\right) \\
& =\frac{1}{n^{2 p}} E\left(\left|\sum_{i=1}^{n} V_{i}\right|^{2 p}\right) \\
& \leq C \frac{1}{n^{2 p}} \max \left(n E\left(\left|V_{1}\right|^{2 p}\right), n^{p}\left(E\left(V_{1}^{2}\right)\right)^{p}\right) \\
& \leq C \frac{1}{n^{2 p}} \times n^{p} \leq C \frac{1}{n^{p}} \leq C\left(\frac{\ln (n)}{n}\right)^{p} .
\end{aligned}
$$

Investigation of (C2). With the same random variables $V_{1}, \ldots, V_{n}$ defined as above, using $2^{j d} \leq 2 n / \ln (n)$, note that $\left|V_{1}\right| \leq 2 \sup _{\mathbf{x} \in[0,1]^{d}}\left|\Psi_{j, \mathbf{k}, u}(\mathbf{x})\right| \leq C 2^{j d / 2} \leq C \sqrt{n / \ln (n)}$. It follows from the Bernstein inequality (see Appendix) with $v=(\kappa / 2) \sqrt{n \ln (n)}, M=C \sqrt{n / \ln (n)}$ and, by (26), with $p=1$, $E\left(V_{1}^{2}\right) \leq C$, that

$$
\begin{aligned}
& P\left(\left|\widehat{d}_{j, \mathbf{k}, u}-d_{j, \mathbf{k}, u}\right| \geq \frac{\kappa}{2} \sqrt{\frac{\ln (n)}{n}}\right) \\
& \quad=P\left(\frac{1}{n}\left|\sum_{i=1}^{n} V_{i}\right| \geq \frac{\kappa}{2} \sqrt{\frac{\ln (n)}{n}}\right)=P\left(\left|\sum_{i=1}^{n} V_{i}\right| \geq v\right) \\
& \quad \leq 2 \exp \left(-\frac{v^{2}}{2\left(n E\left(V_{1}^{2}\right)+v M / 3\right)}\right) \\
& \quad \leq 2 \exp \left(-\frac{((\kappa / 2) \sqrt{n \ln (n)})^{2}}{2 C(n+(\kappa / 2) \sqrt{n \ln (n)} \sqrt{n / \ln (n)} / 3)}\right) \\
& \leq 2 n^{-\theta(\kappa)},
\end{aligned}
$$

where $\theta(\kappa)=\kappa^{2} /(8 C(1+\kappa / 6))$. Taking $\kappa$ such that $\theta(\kappa)=p$, we obtain

$$
\begin{aligned}
& P\left(\left|\hat{d}_{j, \mathbf{k}, u}-d_{j, \mathbf{k}, u}\right| \geq \frac{\kappa}{2} \sqrt{\frac{\ln (n)}{n}}\right) \leq C n^{-p} \\
& \quad \leq C\left(\frac{\ln (n)}{n}\right)^{p} .
\end{aligned}
$$

It follows from Theorem A.3 that

$$
S=E\left(\int_{[0,1]^{d}}|\widehat{f}(\mathbf{x}, \mathbf{m})-f(\mathbf{x}, \mathbf{m})|^{p} d \mathbf{x}\right) \leq C \Theta_{n} .
$$

Upper Bound for $T$. For any $i \in\{1, \ldots, n\}$, set $V_{i}=\mathbf{1}_{\left\{\mathbf{Y}_{i}=\mathbf{m}\right\}}$ $\rho_{\mathrm{m}}$. Then $V_{1}, \ldots, V_{n}$ are $n$ zero-mean iid random variables with $\left|V_{1}\right| \leq 2$. It follows from the Rosenthal inequality (see Appendix) that

$$
\begin{aligned}
T & =E\left(\left|\hat{\rho}_{\mathbf{m}}-\rho_{\mathbf{m}}\right|^{p}\right)=E\left(\left|\frac{1}{n} \sum_{i=1}^{n} V_{i}\right|^{p}\right) \\
& =\frac{1}{n^{p}} E\left(\left|\sum_{i=1}^{n} V_{i}\right|^{p}\right) \\
& \leq C \frac{1}{n^{p}} \max \left(n E\left(\left|V_{1}\right|^{p}\right), n^{p / 2}\left(E\left(V_{1}^{2}\right)\right)^{p / 2}\right) \\
& \leq C \frac{1}{n^{p}} \times n^{p / 2} \leq C \frac{1}{n^{p / 2}} .
\end{aligned}
$$

Combining (22), (30), and (31), we obtain

$$
\begin{aligned}
& E\left(\int_{[0,1]^{d}}|\widehat{g}(\mathbf{x}, \mathbf{m})-g(\mathbf{x}, \mathbf{m})|^{p} d \mathbf{x}\right) \leq C(S+T) \\
& \quad \leq \max \left(\Theta_{n}, n^{-p / 2}\right) \leq C \Theta_{n} .
\end{aligned}
$$

This completes the proof of Theorem 1.

\section{Appendix}

Here we state the two results that have been used for proving our theorem.

Lemma A.1 (see [22]). Let $n$ be a positive integer, let $p \geq 2$, and let $V_{1}, \ldots, V_{n}$ be $n$ zero-mean iid random variables such that $E\left(\left|V_{1}\right|^{p}\right)<\infty$. Then there exists a constant $C>0$ such that

$$
\begin{aligned}
& E\left(\left|\sum_{i=1}^{n} V_{i}\right|^{p}\right) \\
& \leq C \max \left(n E\left(\left|V_{1}\right|^{p}\right), n^{p / 2}\left(E\left(V_{1}^{2}\right)\right)^{p / 2}\right) .
\end{aligned}
$$

Lemma A.2 (see [23]). Let $n$ be a positive integer and let $V_{1}, \ldots, V_{n}$ be $n$ iid zero-mean independent random variables 
such that there exists a constant $M>0$ satisfying $\left|V_{1}\right| \leq M$. Then, for any $v>0$,

$$
P\left(\left|\sum_{i=1}^{n} V_{i}\right| \geq v\right) \leq 2 \exp \left(-\frac{v^{2}}{2\left(n E\left(V_{1}^{2}\right)+v M / 3\right)}\right)
$$

Theorem A.3. We consider a general statistical nonparametric framework. Let $p \geq 1$ and let $f \in \mathbb{L}_{\max (p, 2)}\left([0,1]^{d}\right)$ be an unknown function to be estimated from $n$ observations and consider the wavelet decomposition given by (4). Let $\widehat{c}_{j, \mathbf{k}}$ and $\widehat{d}_{j, \mathbf{k}}$ be estimators of $c_{j, \mathbf{k}}$ and $d_{j, \mathbf{k}}$, respectively, such that there exist two constants $C>0$ and $\kappa>0$ satisfying Assumptions (C1) and (C2) below.

(C1) For any $k \in \mathbf{D}_{\tau}$,

$$
E\left(\left|\widehat{c}_{\tau, \mathbf{k}}-c_{\tau, \mathbf{k}}\right|^{2 p}\right) \leq C\left(\frac{\ln (n)}{n}\right)^{p}
$$

and for any $j \geq \tau$ such that $2^{j d} \leq n, u \in\left\{1, \ldots, 2^{d}-1\right\}$, and $k \in \mathbf{D}_{j}$,

$$
E\left(\left|\widehat{d}_{j, \mathbf{k}, u}-d_{j, \mathbf{k}, u}\right|^{2 p}\right) \leq C\left(\frac{\ln (n)}{n}\right)^{p} .
$$

$$
\Theta_{n}= \begin{cases}\left(\frac{\ln (n)}{n}\right)^{s p /(2 s+d)}, & \text { for } 2 r s>d(p-r), \\ \left(\frac{\ln (n)}{n}\right)^{(s-d(1 / r-1 / p)) p /(2 s-2 d / r+d)}, & \text { for } 2 r s<d(p-r), \\ \left(\frac{\ln (n)}{n}\right)^{(s-d(1 / r-1 / p)) p /(2 s-2 d / r+d)}(\ln (n))^{(p-2 r / q)_{+}}, & \text {for } 2 r s=d(p-r) .\end{cases}
$$

Theorem A.3 can be proved using similar arguments to [16, Theorem 5.1] for a bound of the $\mathbb{L}_{p}$-risk and the multidimensional framework of [17, Theorem 1] for the determination of the rates of convergence.

\section{Competing Interests}

The authors declare that they have no competing interests.

\section{References}

[1] Q. Li and J. S. Racine, Nonparametric Econometrics: Theory and Practice, Princeton University Press, Princeton, NJ, USA, 2007.

[2] N. Akakpo and C. Lacour, "Inhomogeneous and anisotropic conditional density estimation from dependent data," Electronic Journal of Statistics, vol. 5, pp. 1618-1653, 2011.

[3] G. Chagny, "Warped bases for conditional density estimation," Mathematical Methods of Statistics, vol. 22, no. 4, pp. 253-282, 2013.
(C2) For any $j \geq \tau$ such that $2^{j d} \leq n / \ln (n), u \in\left\{1, \ldots, 2^{d}-\right.$ $1\}$, and $k \in \mathbf{D}_{j}$,

$$
P\left(\left|\widehat{d}_{j, \mathbf{k}, u}-d_{j, \mathbf{k}, u}\right| \geq \frac{\kappa}{2} \sqrt{\frac{\ln (n)}{n}}\right) \leq C\left(\frac{\ln (n)}{n}\right)^{p} .
$$

Let us define the estimator $\hat{f}$ by

$$
\begin{aligned}
& \widehat{f}(\mathbf{x}) \\
& =\sum_{\mathbf{k} \in \mathbf{D}_{\tau}} \widehat{c}_{\tau, \mathbf{k}} \Phi_{\tau, \mathbf{k}}(\mathbf{x}) \\
& +\sum_{u=1}^{2^{d}-1} \sum_{j=\tau}^{\infty} \sum_{\mathbf{k} \in \mathbf{D}_{j}} \widehat{d}_{j, \mathbf{k}, u} \mathbf{1}_{\left\{\left|\widehat{d}_{j, \mathbf{k}, u}\right| \geq \mathcal{K} \sqrt{\ln (n) / n\}}\right.} \Psi_{j, \mathbf{k}, u}(\mathbf{x}),
\end{aligned}
$$

$\mathbf{x} \in[0,1]^{d}$, where $j_{1}$ is the integer satisfying $n / \ln (n)<2^{j_{1} d} \leq$ $2 n / \ln (n)$.

Suppose that $f \in \mathbf{B}_{r, q}^{s}(M)$ with $M>0, s>d / r, r \geq 1$, and $q \geq 1$. Then there exists a constant $C>0$ such that

$$
E\left(\int_{[0,1]^{d}}|\widehat{f}(\mathbf{x})-f(\mathbf{x})|^{p} d \mathbf{x}\right) \leq C \Theta_{n}
$$

where

[4] Q. Li and J. Racine, "Nonparametric estimation of distributions with categorical and continuous data," Journal of Multivariate Analysis, vol. 86, no. 2, pp. 266-292, 2003.

[5] A. Antoniadis, "Wavelets in statistics: a review (with discussion)," Journal of the Italian Statistical Society Series B, vol. 6, pp. 97-144, 1997.

[6] W. Härdle, G. Kerkyacharian, D. Picard, and A. Tsybakov, Wavelets, Approximation, and Statistical Applications, vol. 129 of Lecture Notes in Statistics, Springer, New York, NY, USA, 1998.

[7] B. Vidakovic, Statistical Modeling by Wavelets, John Wiley \& Sons, New York, NY, USA, 1999.

[8] V. A. Vasiliev, "A truncated estimation method with guaranteed accuracy," Annals of the Institute of Statistical Mathematics, vol. 66, no. 1, pp. 141-163, 2014.

[9] C. Chesneau, I. Dewan, and H. Doosti, "Nonparametric estimation of a two dimensional continuous-discrete density function by wavelets," Statistical Methodology, vol. 18, pp. 64-78, 2014.

[10] I. Daubechies, Ten Lectures on Wavelets, SIAM, Philadelphia, Pa, USA, 1992.

[11] Y. Meyer, Wavelets and Operators, Cambridge University Press, Cambridge University Press, 1992. 
[12] A. Cohen, I. Daubechies, and P. Vial, "Wavelets on the interval and fast wavelet transforms," Applied and Computational Harmonic Analysis, vol. 1, no. 1, pp. 54-81, 1993.

[13] S. Mallat, A Wavelet Tour of Signal Processing: The Sparse Way, Elsevier, Amsterdam, The Netherlands, 3rd edition, 2009, with Contributions from Gabriel Peyré.

[14] D. L. Donoho and I. M. Johnstone, "Ideal spatial adaptation by wavelet shrinkage," Biometrika, vol. 81, no. 3, pp. 425-455, 1994.

[15] D. L. Donoho, I. M. Johnstone, G. Kerkyacharian, and D. Picard, "Density estimation by wavelet thresholding," The Annals of Statistics, vol. 24, no. 2, pp. 508-539, 1996.

[16] G. Kerkyacharian, D. Picard, L. Birgé et al., "Thresholding algorithms, maxisets and well-concentrated bases," Test, vol. 9, no. 2, pp. 283-344, 2000.

[17] B. Delyon and A. Juditsky, "On minimax wavelet estimators," Applied and Computational Harmonic Analysis, vol. 3, no. 3, pp. 215-228, 1996.

[18] P. Ramirez and B. Vidakovic, "Wavelet density estimation for stratified size-biased sample," Journal of Statistical Planning and Inference, vol. 140, no. 2, pp. 419-432, 2010.

[19] J. Fan, "Local linear regression smoothers and their minimax efficiencies," The Annals of Statistics, vol. 21, no. 1, pp. 196-216, 1993.

[20] M. Abbaszadeh, C. Chesneau, and H. Doosti, "Multiplicative censoring: estimation of a density and its derivatives under the $L_{p}$-risk," REVSTAT Statistical Journal, vol. 11, no. 3, pp. 255-276, 2013.

[21] C. Chesneau, I. Dewan, and H. Doosti, "Nonparametric estimation of a quantile density function by wavelet methods," Computational Statistics and Data Analysis, vol. 94, pp. 161-174, 2016.

[22] H. P. Rosenthal, "On the subspaces $\mathbb{L}^{p}(p \geq 2)$ spanned by sequences of independent random variables," Israel Journal of Mathematics, vol. 8, pp. 273-303, 1970.

[23] V. V. Petrov, Limit Theorems of Probability Theory: Sequences of Independent Random Variables, vol. 4 of Oxford Studies in Probability, Clarendon Press, Oxford, UK, 1995. 


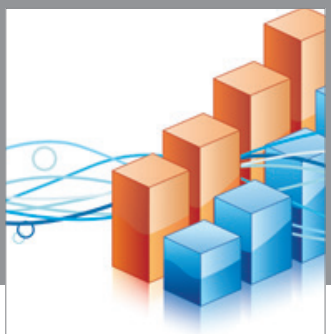

Advances in

Operations Research

vatem alat4

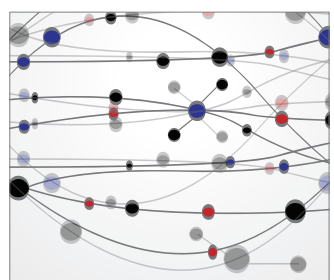

\section{The Scientific} World Journal
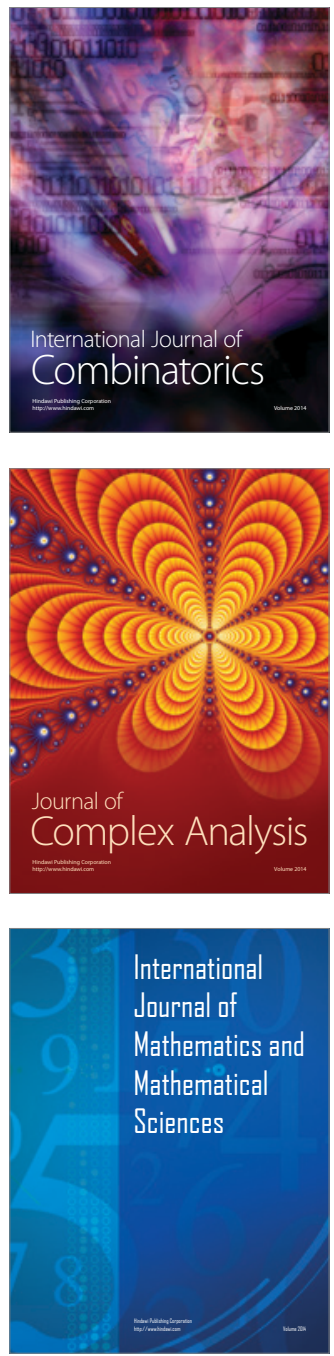
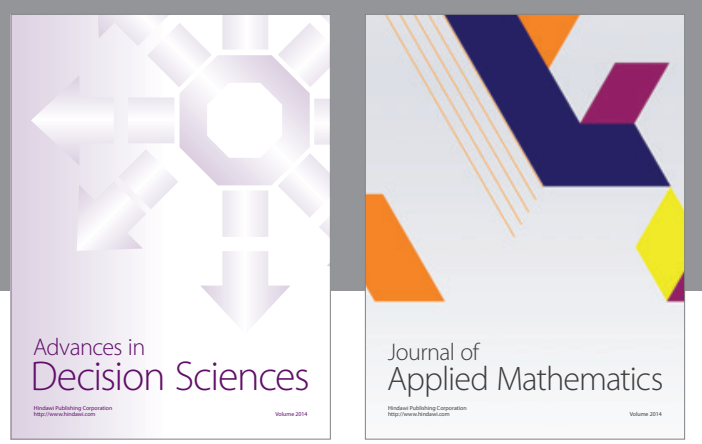

Algebra

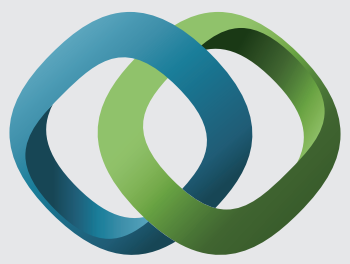

\section{Hindawi}

Submit your manuscripts at

http://www.hindawi.com
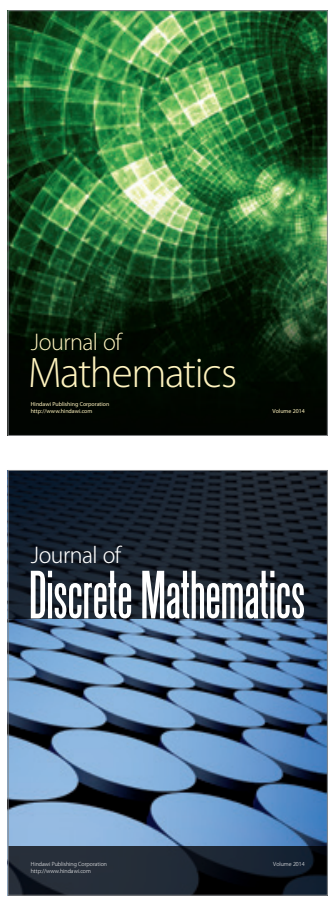

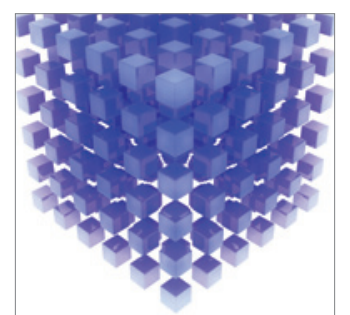

Mathematical Problems in Engineering
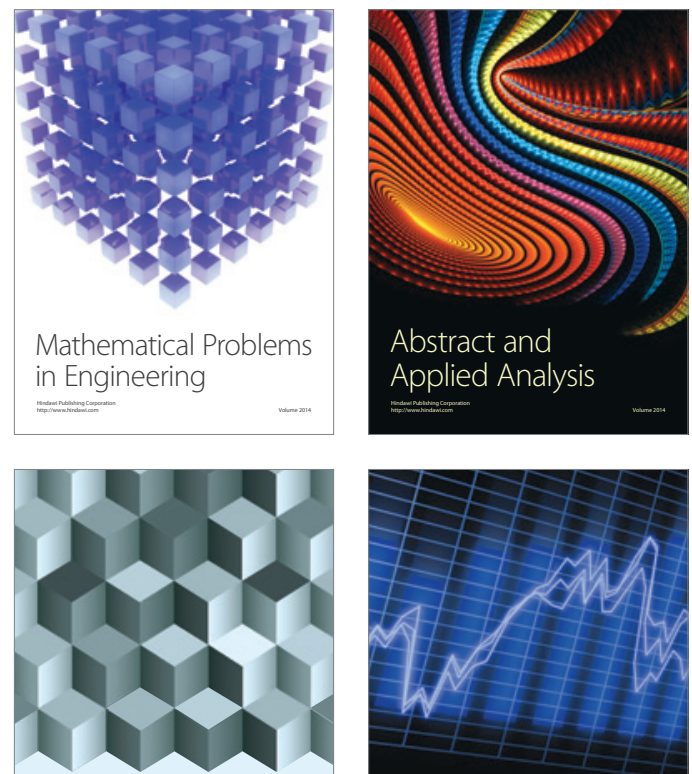

Journal of

Function Spaces

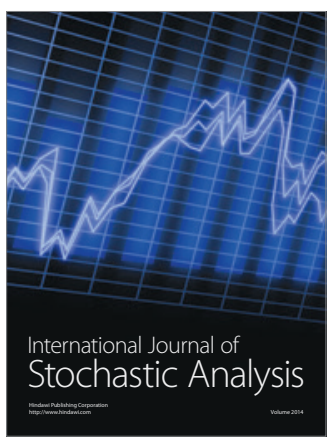

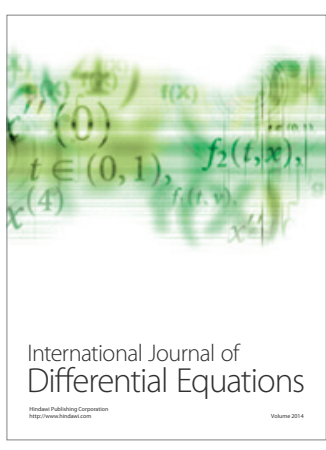
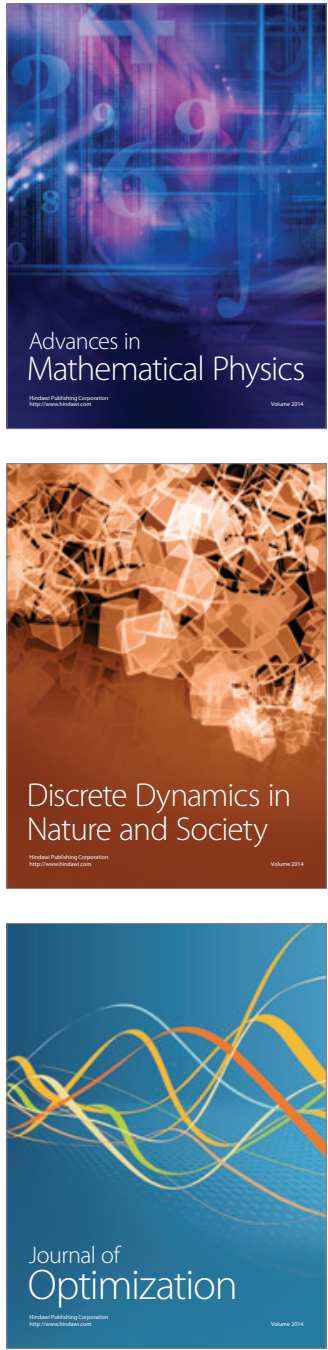